\title{
Efficacy and Tolerability of RAL, MVC and ETV used in combination in the treatment of highly treatment experienced HIV infected patients
}

\author{
Homayoon Khanlou, Shilpa Sayana* \\ From $16^{\text {th }}$ International Symposium on HIV and Emerging Infectious Diseases \\ Marseille, France. 24-26 March 2010
}

\section{Background}

Although the durability of antiretroviral (ARV) efficacy has improved, mainly due to better tolerability, ease of administration (adherence) and potency, some patients still encounter virological and immunological treatment failure. These patients have been on multiple regimens containing nucleoside reverse transcriptase inhibitors (NRTIs), non-nucleoside reverse transcriptase inhibitor (NNRTI) and Protease Inhibitor (PI) and have become resistant to one or more of these classes. Hence, the need for salvage therapies using newly approved antiretrovirals that are NRTI, NNRTI and PI sparing is a clinical reality. Raltegravir, maraviroc and etravirine are 3 new agents with distinguished characteristics which are now available however guidelines for their use continue to evolve and none exist yet on using them all in combination. In this study we reviewed patients who were four ARV class experienced and on salvage regimens consisting of a combination of raltegravir, etravirine and maraviroc.

\section{Methods}

We retrospectively evaluated pts who had documented failure to 3 or 4 different classes of ARVs and were treated with combination of RAL, MVC and ETV. Salvage therapy was prescribed according to: viral tropism, screening genotype and previous resistance tests. Our inclusion criteria was 1) 3 or 4 ARV class experienced, 2) CXCR5 on troFile assay 3) on a regimen containing maraviroc, raltegravir and etravirine together. Data was reviewed and analyzed at baseline and at 24 weeks.

\section{Results}

9 out of 5500 charts fit this inclusion criterion. The mean age was 50 yeras-old (34-62) with majority being male $(7 / 9,78 \%)$. All pts were integrase-inhibitor (RAL) naive and harboring CCR5 tropic virus. The median ETV mutation score was 2.5 (range 0-2.5). The median CD4-cell count and HIV-RNA levels were respectively 200 cells $/ \mathrm{mm}^{3}$ (range: $19-734$ ) and $4.4 \log _{10}$ copies $/ \mathrm{mL}$ (2.3-5.6) at baseline. At week-24 the CD4-cell count was 389 cells $/ \mathrm{mm}^{3}$. The percentage of pts achieving HIVRNA levels $<400 \log _{10}$ copies/mL and $<50 \log _{10}$ copies/ $\mathrm{mL}$ at week 24 were respectively $78 \%(7 / 9)$ and $67 \%(6 /$ 9). In the 2 patients that did not achieve $<400 \log _{10}$ copies/mL HIV-RNA levels poor ARV adherence was documented. All pts tolerated this regimen well with no discontinuation due to adverse events up to 24 weeks.

\section{Discussion}

The combination of RAL, MVC and ETV is safe and highly efficacious in the management of HIV-treatment experienced patients with excellent CD4-cell count recovery and complete viral load suppression with good ARV adherence.

Published: 11 May 2010

doi:10.1186/1742-4690-7-S1-P48

Cite this article as: Khanlou and Sayana: Efficacy and Tolerability of RAL, MVC and ETV used in combination in the treatment of highly treatment experienced HIV infected patients. Retrovirology 2010 7(Suppl 1):P48.

* Correspondence: ssayana@gmail.com

AIDS Healthcare Foundation, Los Angeles, USA 